\title{
Estudio del impacto en la calidad de vida según características demográficas y clínicas en pacientes con rosácea
}

\section{Quality of life in patients with rosacea and its relationship with clinical and demographical characteristics}

\author{
Valeria E. Rossello ${ }^{1}$, Julieta R. Brusa, María N. Andrade, Virginia R. López Gamboa, María S. Gómez \\ Zanni, Mariana B. Papa.
}

1 Universidad Católica de Córdoba. Clínica Universitaria Reina Fabiola, Servicio de Dermatología.

Correspondencia: Rossello Valeria E. Servicio de Clínica Universitaria Reina Fabiola Oncativo 1248-X5004FHP-Cordoba, Argentina; email:

vale_r19@hotmail.com

\section{Resumen}

INTRODUCCIÓN: La rosácea es una enfermedad inflamatoria crónica que afecta mayormente el área centrofacial y se manifiesta clínicamente por la presencia de eritema transitorio o persistente, pápulas y pústulas, en algunas ocasiones llegando a establecerse edema dérmico con formación de fimas; y se clasifica en diferentes subtipos según la severidad y clínica. Es conocido el impacto que produce en la calidad de vida de los pacientes según estudios realizados en distintos países. Sin embargo, se conocen pocos datos en Latinoamérica, particularmente en Argentina.

OBJETIVOS: Determinar el impacto de la rosácea en la calidad de vida de los pacientes atendidos en la Clínica Universitaria Reina Fabiola (CURF) en el año 2018. Describir las características demográficas. Correlacionar la calidad de vida con el subtipo, el grado de severidad de la rosácea y las características demográficas.

MATERIALES Y MÉTODOS: Se realizó un estudio observacional, transversal, analítico. Se incluyeron todos los pacientes que asistieron al Servicio de Dermatología en el año 2018 con diagnóstico clínico de rosácea. Se incluyeron pacientes con diagnóstico clínico de formas clásicas de rosácea, según la clasificación del Grupo Ibero Latinoamericano de Estudio de la Rosácea (GILER), con afectación exclusivamente facial, en pacientes mayores de 16 años. Se analizaron las variables: edad, sexo, fototipo, ocupación según fotoexposición, tratamiento, calidad de vida, subtipo de rosácea, severidad de la rosácea. Para evaluar el impacto en la calidad de vida se utilizó el Dermatology Life Quality Index (DLQI). Para evaluar los subtipos y severidad de la rosácea se utilizó la clasificación de GILER. La relación entre las categorías del DLQI con las variables categóricas nominales se analizaron con test Chi cuadrado y para las variables continuas se utilizó el test de ANOVA. Se consideró significativo un valor de $\mathrm{p} \leq 0.05$.

RESULTADOS: Se incluyeron 72 pacientes. La mayoría (52.8\%) presentó subtipo eritematotelangiectásico (ET), con predominancia del sexo femenino, edad media de $39.2+13.5$ años, fototipo II y III, actividad laboral bajo techo y no habían recibido tratamiento previo. Según el grado de gravedad GILER, la mayoría de los pacientes tuvieron afección moderada (54.2\%). En relación a la calidad de vida evaluada por DLQI, se observó que la mayoría tenía afectación leve (6:3.5) (48.6\%), seguido de afectación moderada (6:8.1) (19.4\%), grave (6:11.2) (13.9\%), muy grave (6:21.0) (1.4\%), mientras que el $16.7 \%$ no presentó afectación de su calidad de vida (6:0.5). No se encontró relación entre el DLQI con el subtipo de rosácea, la severidad de la rosácea y las variables demográficas. 
CONCLUSIONES: En nuestro estudio, la rosácea se presentó predominantemente en el sexo femenino, con fototipo II-III y se observó con mayor frecuencia el subtipo ET. El grado de severidad según GILER en su mayoría fue moderado. La rosácea afectó levemente la calidad de vida en la mayoría de los pacientes. No se encontró relación estadísticamente significativa entre el DLQI con los subtipos y severidad de la rosácea, como así tampoco con las variables demográficas. Estos datos coinciden en muchos aspectos con la literatura consultada.

Palabras claves: Rosácea, Calidad de vida.

\section{Abstract}

INTRODUCTION: Rosacea is a chronic inflammatory disease that mostly affects the mid facial area and is manifested clinically by the presence of transient or persistent erythema, papules and pustules, on some occasions dermal edema with fima formation is established. It is classified in different subtypes according to the severity and clinical characteristics. The impact of this disease on the quality of life of patients has been studied in different countries; however, there is scarse data regarding this issue in Latin America, particularly in Argentina.

OBJECTIVES: To determine the impact of rosacea on the quality of life of patients at the Reina Fabiola University Clinic in 2018. To describe the demographic characteristics of patients with rosacea. To analyze the variables associated with quality of life.

MATERIALS AND METHODS: This is an observational, cross-sectional, analytical study. We included all patients with diagnosis of rosacea who attended the Dermatology Service in 2018, who had classic forms of rosacea (according to the GILER), with exclusively facial involvement and in patient older than 16 years old. We analyzed the following variables: age, sex, phototype, occupation according to sun exposure, previous treatment, quality of life as per the Dermatology Life Quality Index (DLQI), subtype and severity of rosacea. The relationship between the DLQI categories with the categorical nominal variables was analyzed with Chi-square test and for the continuous variables the ANOVA test was used. A p value $\leq 0.05$ was considered significant.

RESULTS: The final sample consisted of a total of 72 patients; the majority being females, with a mean age of $39.2+13.5$ years. The majority $(52.8 \%)$ of the patients presented the erythematous telangiectatic (ET) subtype of rosacea, phototype II and III, indoor work activity and stated they had not received previous treatment. According to the GILER classification, most of the patients had a moderate severity of the disease (54.2\%). The DLQI Regarding the DLQI, the majority had mild involvement (6:3.5) $(48.6 \%)$, followed by moderate involvement involvement $(6: 8.1)(19.4 \%)$, severe involvement, $(6: 11.2)$ $(13.9 \%)$ and only few patients had very severe involvement $(6: 21.0)(1.4 \%)$. Sixteen percento of the patients had no impact on their quality of life (6:0.5). We found no relationship between the DLQI with the different subtypes of rosacea, the severity of rosacea and demographic variables.

CONCLUSION: In this study, rosacea occurred predominantly in females, mostly with phototype II-III. The most frequent clinical subtype was ET and the severity according to GILER was mostly moderate. No statistically significant relationship was found between the DLQI with the clinical subtypes and severity of rosacea, as well as with demographic variables. These data is coincident coincide in many aspects with the published literature.

Keywords: Rosacea, Quality of life.

\section{Introducción}

La rosácea es una enfermedad inflamatoria crónica $^{1-8}$ de afección predominante en el área centrofacial $^{3-5,7}$ que cursa con exacerbaciones y remisiones ${ }^{9}$, por lo que es un motivo frecuente de consulta dermatológica. Actualmente, se estima una prevalencia del 2-10\% según estudios realizados en Europa ${ }^{1,3,4}$. En Latinoamérica la estadística de esta patología no ha sido ampliamente estudiada; a pesar de ello se reportó una prevalencia de $2 \%$ en Perú ${ }^{10}$. Así mismo, se conoce que la incidencia está aumentando progresivamente ${ }^{9}$. Es más frecuente en foto tipos bajos (Fitzpatrick $\mathrm{I}_{-} \mathrm{II}^{4}$ ), caucásicos de ascendencia celta $^{3,11,12}$, en el sexo femenino y presenta un pico de presentación entre los 30 y 50 años de edad ${ }^{3,4,13}$. La explicación etiológica y fisiopatogénica es multifactorial. Incluye alteraciones del sistema inmune, de los mecanismos de respuesta neurogénica y vascular ${ }^{2-3,10}$. 
Se conoce que existe una predisposición genética $^{3,4,13}$ además de los factores desencadenantes de los brotes ${ }^{4,8}$.

Clínicamente, la rosácea se caracteriza por presentar hallazgos clínicos primarios: eritema (transitorio o persistente), telangiectasias, pápulas y pústulas ${ }^{10-14}$ asociados o no a hallazgos clínicos secundarios como ardor o prurito, placas eritemato-edematosas, xerosis, lesiones extrafaciales, cambios fimatosos y enfermedad oftálmica $^{3-5,10}$. Existen varias clasificaciones ${ }^{8-13}$, pero según el Grupo Ibero Latinoamericano de Estudio de la Rosácea (GILER) ${ }^{5}$ se dividen en cuatro subtipos clásicos: eritemato-telangiectásica (ET), pápulo-pustulosa (PP), fimatosa $(\mathrm{F})$ y ocular. Existen además formas especiales de rosácea como la rosácea infantil y extrafacial ${ }^{5}$. La rosácea granulomatosa no se considera un subtipo sino una variante de rosácea ${ }^{4,5}$. Se conocen además diferentes grados de severidad de rosácea (leve, moderado y severo), los cuales se describen de acuerdo a las características clínicas primarias presentes en los pacientes con rosácea ${ }^{5}$.

Aunque antiguamente se consideraba como una afección puramente estética, actualmente se considera que la rosácea produce un impacto negativo en la calidad de vida de los pacientes. Las alteraciones del aspecto físico, asociadas a un estado pro inflamatorio constante (en el que se teme el momento del brote), causan problemas de autoaceptación $^{4,6,12,13}$. Muchos de los pacientes son vistos como alcohólicos a consecuencia del eritema y el rinofima, por lo cual padecen sentimientos de vergüenza, ansiedad, falta de confianza, baja autoestima, depresión y fobia social $^{11-16}$. Sumado a esto, remarcan como frustrante el hecho de que los profesionales hacen hincapié en el control y no en la curación de la enfermedad $^{16}$. Este sentimiento de subestimación por sus médicos, puede corresponder a que la evaluación clínica no necesariamente refleja la afección psico-social ${ }^{1,7,11,13,15}$. Por este motivo, la medición del índice de salud relacionada con la calidad de vida, es de suma importancia en la práctica clínica ${ }^{3,7}$. En Latinoamérica no hay estudios en relación a la calidad de vida para rosácea por lo que nos parece importante analizar que ocurre en nuestro medio.

\section{Objetivos}

\section{Objetivo principal}

- Determinar el impacto de la rosácea en la calidad de vida de los pacientes atendidos en la Clínica Universitaria Reina Fabiola (CURF) en el año 2018.

\section{Objetivo Secundario}

- Describir la población de la CURF adulta afectada por rosácea en el año 2018 según características demográficas.

- Analizar la relación entre la puntuación del DLQI y el subtipo de rosácea según GILER.

- Analizar la relación entre el DLQI y grado de severidad de la rosácea mediante la clasificación GILER.

- Analizar la relación entre la puntuación del DLQI con las siguientes variables demográficas: edad, sexo, fototipo y ocupación.

\section{Materiales y Métodos}

Diseño del estudio: observacional, transversal, analítico.

$\square$ Población a estudiar: Pacientes que acudieron en forma espontánea al Servicio de Dermatología de la CURF con diagnóstico clínico de rosácea durante el período del 1 de junio al 31 de diciembre del 2018.

$\square$ Muestra:

- Diseño muestral: no probabilístico, consecutivo.

- Universo: del total de pacientes que acudieron al Servicio de Dermatología durante el período determinado, se analizaron todos los que fueron diagnosticados clínicamente con rosácea. La población que acudió regularmente a la CURF pertenece a un estrato social medio y alto y posee cobertura médica privada.

- Recolección de los datos: Se utilizaron los registros de historias clínicas informáticas del Servicio de Dermatología de la CURF, el cuestionario DLQI (Dermatology Life Quality Index) sobre calidad de vida y las planillas de recolección de datos en relación al presente estudio

Criterios de inclusión: pacientes con diagnóstico clínico de formas clásicas de rosácea, de acuerdo a la clasificación de GILER $^{5}$ (Tabla1-2), con afectación exclusivamente facial, mayores de 16 años, con datos clínicos y demográficos en la planilla de estudio completa, que aceptaron participar voluntariamente y que estaban capacitados para responder la encuesta.

Criterios de exclusión: pacientes con enfermedades autoinmunitarias

$\mathrm{y}$ autoinflamatorias.

Variables del estudio:
A. Variables demográficas
- Edad: años
- Sexo: femenino/masculino
- Fototipo: I-IV 
- Ocupación según fotoexposición:

interior/exterior

- Tratamiento:

o Realizó tratamiento: $\mathrm{Si} / \mathrm{No}$

Conforme: $\mathrm{Si} / \mathrm{No}$

B. Variables clínicas

Tabla 1 Clasificación de rosácea según GILER ${ }^{5}$

\begin{tabular}{|c|l|l|}
\hline $\begin{array}{c}\text { Formas Clásicas } \\
\text { Subtipos }\end{array}$ & Formas especiales & \multicolumn{1}{|c|}{ Variante } \\
\hline $\begin{array}{c}\text { 1- Eritemato- } \\
\text { telangiectásica }\end{array}$ & $\begin{array}{l}\text { Rosácea infanti1* } \\
\text { Rosácea } \\
\text { 2- Pápulo- } \\
\text { pustulosa } \\
\text { 3- Fimatosa }\end{array}$ & $\begin{array}{l}\text { Rosácea } \\
\text { granulomatosa* }\end{array}$ \\
4- Ocular* & & \\
\hline
\end{tabular}

*se excluyeron del estudio.

- Calidad de vida: se evaluó mediante el DLQI. (Cuestionario en anexo $1^{17-18}$ validado en español para enfermedades dermatológicas). El cuestionario consta de 10 preguntas, cuyas respuestas tienen puntuación de 0 a 3 según la respuesta. El resultado se obtuvo con la sumatoria de la puntuación, con un máximo de 30 , lo cual se expresa como afectación en la calidad de vida 0-1: No afecta, 2-5: leve, 6-10: moderado,11-20: severo, 21-30: muy grave.

Tabla 2 Grados de severidad de la rosácea según GILER $^{5}$ :

\begin{tabular}{|l|l|l|l|l|}
\hline Severidad & Eritema & Telangiectasias & Pápulas/pústulas & Placas \\
\hline Leve & $\begin{array}{l}\text { Solo } \\
\text { eritema } \\
\text { episódico } \\
\text { (flushing) }\end{array}$ & Ausentes & Aisladas & Ningunas \\
\hline Moderado & $\begin{array}{l}\text { Eritema } \\
\text { episódico } \\
\text { (flushing) } \\
\text { con poco } \\
\text { eritema } \\
\text { persistente }\end{array}$ & $\begin{array}{l}\text { Pocas } \\
\text { (evidentes solo } \\
\text { con } \\
\text { magnificación) }\end{array}$ & Algunas & Ningunas \\
\hline Severo & $\begin{array}{l}\text { Eritema } \\
\text { persistente } \\
\text { intenso }\end{array}$ & $\begin{array}{l}\text { Evidentes a } \\
\text { simple vista y } \\
\text { abundantes }\end{array}$ & Abundantes & Presentes \\
\hline
\end{tabular}

Procesamiento de datos y análisis estadístico

Variables cualitativas categóricas: frecuencias absolutas y relativas.

Variables cuantitativas: medidas de posición y distribución.

La relación entre las categorías del DLQI con las variables categóricas nominales se analizaron con test Chi cuadrado y para las variables continuas se utilizó el test de ANOVA. Se consideró significativo un valor de $\mathrm{p} \leq 0.05$.

\section{Consideraciones éticas}

El estudio se realizó de acuerdo a la Declaración de Helsinki. Se realizó un consentimiento informado para los pacientes que participaron del estudio, garantizando la confidencialidad respecto de la identidad de los mismos y sus datos filiatorios, los cuales fueron registrados de manera codificada. Previo a la recolección de datos el estudio fue evaluado por el Departamento de Docencia e Investigación y por el Comité de Ética institucional. Se respetó la ley 25326 de Hábeas Data y ley 9694 sobre investigación en seres humanos de la provincia de Córdoba.

\section{Resultados}

La muestra final estuvo conformada por un total de 72 pacientes. La edad media fue de $39.2+13.5$ años. Con respecto al sexo, la mayoría $(n=56$, $77.8 \%$ ) de los pacientes fueron de sexo femenino. Los fototipos más frecuentes fueron II y III $(n=32$, $44.4 \%$ en ambos casos), seguidos por fototipo I $(\mathrm{n}=7,9.7 \%)$, siendo el menos frecuente el fototipo IV $(n=1,1.4 \%)$. En la mayoría de los casos, la ocupación laboral de los pacientes fue bajo techo, $(n=63,87.5 \%)$, mientras que el $12.5 \%$ trabajaba al exterior. De los pacientes incluidos en el estudio, $33(45.8 \%)$ pacientes refería haber estado bajo tratamiento, de los cuales la mayoría estaba conforme con los resultados $(\mathrm{n}=25,75.8 \%)$.

Los subtipos de rosácea se distribuyeron de la siguiente manera: la mayoría presentó subtipo ET $(\mathrm{n}=38,52.8 \%)$, seguido por el tipo PP $(\mathrm{n}=32$, $44.5 \%$, ) y el menos frecuente fue el tipo $\mathrm{F}(\mathrm{n}=2$, $2.8 \%$,). Se observó que según el grado de severidad GILER, la mayor parte de los pacientes tenían afección moderada $(n=39,54.2 \%)$, seguidos por leve $(n=24,33.3 \%)$ y la minoría $(n=9,12.5 \%)$ tuvo afección severa.

En relación a la calidad de vida evaluada por DLQI, se observó que la mayoría tenía afectación leve $(6: 3.5)(n=35,48.6 \%)$, seguida por afectación moderada $(6: 8.1)(n=14,19.4 \%)$, severa $(6: 11.2)$ $(\mathrm{n}=10,13.9 \%)$ y solo un paciente $(1.4 \%)$ presentó afección muy severa (6:21.0). El 16.7\% ( $\mathrm{n}=12)$ negó afectación de su calidad de vida (6:0.5).

La relación entre clasificación DLQI con el subtipo de rosácea, la severidad de la rosácea y las variables demográficas no fue estadísticamente significativa (Tabla 3 ). 
Tabla 3. Variables demográficas y clínicas relacionadas con la calidad de vida según el DLQI.

\begin{tabular}{|c|c|c|c|c|c|c|c|}
\hline \multicolumn{8}{|c|}{ Grado de afectación de calidad de vida según DLQI \%(n) } \\
\hline & & $\begin{array}{l}\text { Leve } \\
(35)\end{array}$ & $\begin{array}{l}\text { Moderado } \\
\text { (14) }\end{array}$ & $\begin{array}{c}\text { Severo } \\
(10)\end{array}$ & $\begin{array}{c}\text { Muy } \\
\text { severo } \\
\text { (l) }\end{array}$ & $\begin{array}{c}\text { Sin } \\
\text { Afectación } \\
(12)\end{array}$ & $\begin{array}{l}\text { Valor } \\
\text { dep }\end{array}$ \\
\hline \multirow{3}{*}{ Sexo } & & & & & & & 0.1940 \\
\hline & $\begin{array}{l}\text { Femenino } \\
\text { (n:56) }\end{array}$ & $52(29)$ & $18(10)$ & $16(9)$ & $14(8)$ & 0 & \\
\hline & $\begin{array}{l}\text { Masculino } \\
(\mathrm{n}: 16)\end{array}$ & $38(6)$ & $31(4)$ & 6(1) & 6(1) & $25(4)$ & \\
\hline \multirow{2}{*}{$\begin{array}{c}\text { Edad } \\
\text { (añosi) }\end{array}$} & & & & & & & 0.0507 \\
\hline & & $37 \pm 12.5$ & $43.9 \pm 15.8$ & $32.1 \pm 7.92$ & $30 \pm 0$ & $46.5 \pm 15.4$ & \\
\hline \multirow{5}{*}{ Fototipo } & & & & & & & 0.6324 \\
\hline & I $\quad(n: 7)$ & $29(2)$ & $43(3)$ & 0 & 0 & $29(2)$ & \\
\hline & II $\quad(n: 32)$ & $47(15)$ & $13(4)$ & $22(7)$ & 0 & $19(6)$ & \\
\hline & III $(\mathrm{n}: 32)$ & $53(17)$ & $22(7)$ & $9(3)$ & $1(1)$ & $13(4)$ & \\
\hline & IV (n:1) & $100(1)$ & 0 & 0 & 0 & 0 & \\
\hline \multirow{3}{*}{ Ocupación } & & & & & & & 0.8476 \\
\hline & $\begin{array}{l}\text { Interior } \\
(\mathrm{n}: 63)\end{array}$ & $51(32)$ & 19 (12) & $13(1)$ & $1(8)$ & $16(10)$ & \\
\hline & $\begin{array}{l}\text { Exterior } \\
(\mathrm{n}: 9)\end{array}$ & $33(3)$ & $22(2)$ & $22(2)$ & 0 & $22(2)$ & \\
\hline \multirow{4}{*}{$\begin{array}{c}\text { Tipo } \\
\text { de } \\
\text { rosácea }\end{array}$} & & & & & & & 0.1251 \\
\hline & ET $(n: 38)$ & $50(19)$ & $13(5)$ & $13(5)$ & 0 & $24(9)$ & \\
\hline & PP (n:32) & $50(16)$ & $22(7)$ & $16(5)$ & $3(1)$ & $9(3)$ & \\
\hline & F $\quad(\mathrm{n}: 2)$ & $50(36)$ & $22(16)$ & $16(11)$ & $3(2)$ & $9(7)$ & \\
\hline \multirow{4}{*}{$\begin{array}{l}\text { Severidad } \\
\text { (GILER) }\end{array}$} & & & & & & & 0.2237 \\
\hline & $\begin{array}{l}\text { Leve } \\
(\mathrm{n}: 24)\end{array}$ & 50 (12) & $13(3)$ & $21(5)$ & 0 & $17(4)$ & \\
\hline & $\begin{array}{l}\text { Moderado } \\
\text { (n:39) }\end{array}$ & $50(20)$ & $15(6)$ & $13(5)$ & $3(1)$ & $18(7)$ & \\
\hline & $\begin{array}{l}\text { Severo } \\
(\mathrm{n}: 9)\end{array}$ & $33(3)$ & $56(5)$ & 0 & 0 & 11(1) & \\
\hline
\end{tabular}

DLQI: Índice de Calidad de Vida Dermatológica. GILER: Grupo Ibero Latinoamericano de Estudio de la Rosácea. ET: eritemato-telangiectásica. F: fimatosa. PP: pápulo-pustulosa.

\section{Discusión}

Este estudio evidenció que la rosácea afecta en forma negativa la calidad de vida de los pacientes, en la mayoría de los casos de manera leve $(48.6 \%)$. Además, se evidenció que, en la población estudiada, la rosácea es más frecuente en la cuarta década de la vida, en fototipos II y III $(88.8 \%)$, en pacientes que trabajan bajo techo y la mayoría está conforme con los tratamientos recibidos.

Las publicaciones revisadas evidencian un rango entre 42-52.6 años ${ }^{7,11,12,14,19,20}$. Lo cual coincide con nuestro resultado, donde los pacientes presentan una edad de $39.2+13.5$ años, ligeramente más temprano que en la bibliografía. De los estudios revisados, la mayoría de los pacientes que participaron fueron de sexo femenino $^{6,7,11,12,16,19-21}$, lo cual coincide con nuestro trabajo. En cuanto a los fototipos solo fueron descriptos en dos estudios: en el estudio de Aksoy et al16 donde los más frecuentemente reportados fueron los fototipos II y III coincidiendo con nuestros resultados; sin embargo, en el estudio realizado por $\mathrm{Wu}$ et $\mathrm{al}^{16}$ fueron los fototipos III y IV. Consideramos que estos datos pueden tener relación con las etnias involucradas.

En cuanto a los subtipos de rosácea fueron pocos estudios los que los evaluaron. Belilovsky et $\mathrm{al}^{22}$ describió que el subtipo más frecuente fue ET seguido de PP, lo cual es compatible con nuestros resultados, mientras que Hiltscher et $\mathrm{al}^{23}$ hallaron que la forma más frecuente fue PP, seguido de F. La afectación en la calidad de vida según la bibliografía, es muy variada. Los estudios que usan DLQI, informan rangos muy amplios, con valores entre 4.1(afectación leve) a 17.3 (afectación severa) ${ }^{1}$. Nuestro estudio demostró que la mayoría de los pacientes tienen afectación leve de la calidad de vida, lo cual condice con los resultados de Beikert et $\mathrm{al}^{11}$, Bóhm et $\mathrm{al}^{14}$, Langerbrunch et $\mathrm{al}^{19}$. Mientras que $\mathrm{Wu}$ et $\mathrm{al}^{6}$, Bewley et al7, Li et al ${ }^{15}$, Aksoy et al ${ }^{16}$, tuvieron mayor porcentaje de pacientes con afectación moderada y grave.

En cuanto a la relación de la calidad de vida y las variables demográficas y clínicas, los datos publicados son contradictorios. Acerca de calidad de vida y sexo, en el estudio de $\mathrm{Wu}$ et $\mathrm{al}^{16}$ no se demostraron diferencias significativas al igual que en nuestro trabajo. En cambio, en el estudio de Aksoy et $\mathrm{al}^{16}$ y Langenbrunch et $\mathrm{al}^{19}$ las mujeres tenían peor calidad de vida y en el estudio de Böhm et al $^{14}$ el más afectado fue el sexo masculino. Sobre la relación de la calidad de vida con edad, en tres estudios se encontraron que a menor edad fue peor la calidad de vida ${ }^{11,14,16}$. En nuestro estudio si bien la relación no fue estadísticamente significativa, se observó una tendencia a una peor calidad de vida en pacientes más jóvenes. En relación a la calidad de vida con los subtipos de rosácea, en el estudio de Aksoy et al $^{16}$ no se encontraron diferencias significativas lo cual concuerda con nuestro estudio. Böhm et $\mathrm{al}^{14}$ al igual que Aksoy et $\mathrm{al}^{16}$, en sus reportes correlacionan la calidad de vida con la severidad de la rosácea, medidas que no pueden extrapolarse a nuestro trabajo, ya que fueron evaluadas con otra metodología.

Las limitaciones de este estudio están condicionadas por el tamaño de la muestra, la variabilidad interobservador en fototipos $y$ severidad y por el subgrupo de pacientes correspondientes al estrato social de la Institución donde se llevó a cabo la investigación. Expandir la muestra en número de pacientes y/o instituciones permitiría a futuro un estudio más heterogéneo, ofreciendo la posibilidad de datos estadísticos más representativas de nuestra población.

\section{Conclusión}

En la población estudiada, la rosácea afectó levemente la calidad de vida de los pacientes, predominó en el sexo femenino, pacientes con fototipo II-III, siendo el tipo ET la forma clínica de mayor frecuencia de presentación. El grado de severidad según GILER en su mayoría fue moderado. No se encontró una relación 
estadísticamente significativa entre el DLQI con el subtipo, la severidad de la rosácea, como así tampoco con el sexo, la edad, el fototipo, ocupación ni tratamiento previo realizado. Argentina carece de investigaciones sobre calidad de vida en pacientes con rosácea, ya que no se han encontrado en la literatura nacional estudios científicos que así lo demuestren, por lo que nuestros datos podrían representar el puntapié inicial de futuras investigaciones.

\section{Bibliografía}

1. Van der Linden MM, Van Rapard DC, Damms JC, Sprangers MA, Spuls PI,de Korte J. Health related quality of life in patient with cutaneous rosacea: a systematic review. Acta derm veneareol 2015; 95(4):395-400.

2. Holmes AD, Steinhoff M. Integrative concepts of rosacea pathophysiology,clinical presentation an new therapeutics. Exp Dermatol 2017; 26(8):659-667.

3. Troielli P, González OFM, Ríos YJM, Vázquez MOT, Pabón MJG, Ibarra Fernández BR, Quiñones VR, Barona MI, Piancastelli A, Larralde M, Alcalá PD, Costa ALM, Meléndez E, Molina CMT, Torezan L, Torres LV. Actualización y recomendaciones para el diagnóstico y tratamiento de la rosácea en Latinoamérica. Med Cutan Iber Lat Am 2016; 44 (S1): S7-S26.

4. Barco D, Alomar A. Rosácea. Actas Dermosifiliogr. 2008; 99:244-56.

5. Kaminsky A, Flórez WM, Piquero MJ, Herane MI, Diez MJC, Raimondo N, Bagatin E, Steiner D, Zegpi E, Rivera M, Madrigal F, Guerra A, del Ojo D,Adame G, Arias I, Ferro L, Gómez M, Moreno J, Cantilo L, Maciel R,Sandoval B, del Solar M, Giansante E, Pérez AR. Informe de Consenso IberoLatinoamericano 2016 sobre la clasificación clínica y terapéutica de la rosácea. Med Cutan Iber Lat Am 2016; 44 (1) :6-10

6. Wu Y, Fu C, Zhang W, Li C, Zhang J. The dermatology life quality index (DLQI) and the hospital anxiety and depression (HADS) in Chinese rosácea patients. Psychol Health Med. 2018; 23(4):369-374.

7. Bewley A, Fowler J, Schöfer H, Kerrouche N, Rives V. Erythema of Rosacea Impairs Health-Related Quality of Life: Results of a Meta-analysis. Dermatol Ther (Heidelb). 2016; 6(2):237-47.

8. Gil Díaz MJ, Boixeda de Miguel JP, Truchuelo Díez M, Morais-Cardoso P.
Rosácea: revisión y nuevas alternativas terapéuticas. Semergen 2011; 37:83-6.

9. Kim J, Ahn JW, Ha S, Kwon SH, Lee O, Oh C. Clinical assessment of rosácea severity: oriental score vs. quantitative assessment method with imaging and biomedical tolos. Skin Res Technol. 2017; 23(2):186-193.

10. Barona MI, Orozco B, Motta A, Meléndez E, Flaminio Rojas R, Pabón JG,Balcázar LF. Rosácea: actualización. Piel 2015; 30:485502.

11. Beikert FC, Langenbruch AK, Radtke MA, Augustin M. Willingness to pay and quality of life in patients with rosacea. J Eur Acad Dermatol Venereol. 2013; 27(6):734-8.

12. Menezes N, Moreira A, Mota G, Baptista A. Quality of life and rosacea: Pulsed dye laser impact. J Cosmet Laser Ther. 2009; 11(3):139-41.

13. Tu T Huynh, Ph D. Burden of Disease: The Psychosocial Impact of Rosacea on a Patient's Quality of Life. Am Health Drug Benefits. 2013; 6(6):348-354.

14. Böhm D, Schwanitz P, Stock Gissendanner S, Schmid-Ott G, Schulz W. Symptom severity and psychological sequelae in rosacea: Results of a survey. Psichol Healt Med. 2014; 19(5):586-91.

15. Li J, Li M, Chen Q, Fu J, Zhang M, Hao F. Quality of life among patients with rosacea: an investigation of patients in China using two structured questionnaires. J Eur Acad Dermatol Venereol 2015;30(10): e98-e99.

16. Aksoy B, Altaykan-Hapa A, Egemen D, Karagöz F, Atakan N. The impact of rosacea on quality of life: effects of demographic and clinical characteristics and various treatment modalities. Br J Dermatol. 2010; 163(4):71925.

17. Basra MK, Fenech R, Gatt RM, Salek MS, Finlay AY. The Dermatology Life Quality Index 1994-2007: a comprehensive review of validation data and clinical results. $\mathrm{Br} \mathrm{J}$ Dermatol. 2008; 159(5):997-1035.

18. Finlay AY, Khan GK. Cuestionario sobre la calidad de vida- Dermatología. http://www.solapso.org/archivos/evaluacion/ DLQI-es.pdf. Descarga: 31-10-2016.

19. Langerbrunch AK, Beket E, Augustin M. Quality of health care of rosacea in Germany from the patient's perspective: results of the national health care study Rosaral 2009. Dermatology. 2011;223(2):124-30. 
20. Shim TN, Abdullah A. The effect of pulsed dye laser on the dermatology life quality index in erythematotelangiectatic rosacea patients: an assessment. J Clin Aesthet Dermatol. 2013; 6 (4):30-2.

21. Tan J, Steinhoff M, Bewley A, Gierler U and Rives V. Characterizing high-burden rosacea subjects: A multivariate risk factor analysis from a global survery. J Dermatolog Trear 2019; 23:1-27.

22. Belilovsky C, Ilhe F, Pernet AM. Equale study: impact of rosacea on quality of life of affected patients. J Am Acad Dermatol. 2007;56(2): AB16.

23. Hiltscher D, Boslet W.T, Fuchslocher M, Sinkgraven R, Rzany B. Lebensqualität bei Patienten mit Rosacea and Rhinophym. Ajt Dermatol 2001; 27(12):391-394.

$$
\text { (C) (1) \&(0) }
$$

